\title{
Binary system delays and timing noise in searches for gravitational waves from known pulsars
}

\author{
Matthew Pitkin and Graham Woan ${ }^{1}$ \\ ${ }^{1}$ Department of Physics and Astronomy, University of Glasgow, University Avenue, Glasgow, G12 8QQ, UK⿵
}

(Dated: September 17, 2018)

\begin{abstract}
The majority of fast millisecond pulsars are in binary systems, so that any periodic signal they emit is modulated by both Doppler and relativistic effects. Here we show how well-established binary models can be used to account for these effects in searches for gravitational waves from known pulsars within binary systems. A seperate issue affecting certain pulsar signals is that of timing noise and we show how this, with particular reference to the Crab pulsar, can be compensated for by using regularly updated timing ephemerides.
\end{abstract}

PACS numbers: 97.60.Gb, 04.80.Nn, 07.05.Kf

\section{INTRODUCTION}

Neutron stars are thought to be strong candidates for the emission of detectable continuous gravitational waves [1], including the 1627 pulsars currently discov$\operatorname{ered}^{1}$. The majority of these pulsars have been discovered through dedicated radio surveys. Surveys are ongoing, but estimates of the number of active pulsars in the galaxy can be made by inference from the current population, taking into account biasing from selection effects, and the supernova rate. Estimates give values of $\sim 200000$ active pulsars within our galaxy (see Ref. [4]).

Pulsars are found in a wide range of environments. Some are directly associated with the supernova remnants (SNRs) in which they were born. These are typically young pulsars whose birth velocity has not yet caused a large displacement from the remnant and the SNR has not dissipated into the interstellar medium (ISM). Some pulsars are found in binary systems as the companions of a wide range of astronomical bodies from planets, through main sequence stars, to white dwarfs and other neutron stars. The fastest, 'millisecond', pulsars (pulsars with rotation periods of $<10 \mathrm{~ms}$ ) are usually found within binary systems, and often within globular clusters, their rapid rotation rate a consequence of being spun-up by accretion of material from a stellar companion [5]. Pulsars are also seen without any association, and in this paper we will classify any pulsar not in a binary system as isolated.

Pulsars are generally seen to spin-down as they lose rotational energy through a variety of emission mechanisms, but the primary loss mechanism is thought to be magnetic dipole radiation. Other potential mechanisms include particle acceleration and gravitational radiation. Whatever the mechanisms at work, the rotational phase evolution of a pulsar can generally be well described by

\footnotetext{
${ }^{a}$ Electronic address: matthew@astro.gla.ac.uk

1 As given by the Australia Telescope National Facility - ATNF - online pulsar catalogue [2, 3] as of $6^{\text {th }}$ Nov 2006, from which time all subsequent pulsar numbers will be taken.
}

a short Taylor expansion,

$$
\begin{aligned}
\phi(T)= & \phi_{0}+2 \pi\left\{\nu_{0}\left(T-t_{0}\right)+\frac{1}{2} \dot{\nu}_{0}\left(T-t_{0}\right)^{2}\right. \\
& \left.+\frac{1}{6} \ddot{\nu_{0}}\left(T-t_{0}\right)^{3}+\ldots\right\},
\end{aligned}
$$

where $\phi_{0}$ is the initial phase, $\nu_{0}$ and its time derivatives are the pulsar frequency and spin-down coefficients at an epoch $t_{0}$, and $T$ is the time in a frame comoving with the pulsar. For the vast majority of pulsars the value of $\dot{\nu}$ is very small and $\ddot{\nu}$ is unmeasurable or swamped by timing noise (see $₫ \mathrm{IV}$ ). Note that gravitational waves emitted from a triaxial, non-precessing, neutron star come from the quadrupolar component of the rotating body and so will have exactly twice the phase evolution described by Eq. 1.1.

The expected gravitational wave signal from a triaxial neutron star is given by [ $[$ ]

$$
\begin{aligned}
h(t)= & \frac{1}{2} F_{+}(t ; \psi) h_{0}\left(1+\cos ^{2} \iota\right) \cos 2 \phi(t) \\
& +F_{\times}(t ; \psi) h_{0} \cos \iota \sin 2 \phi(t),
\end{aligned}
$$

where $\phi(t)$ is that given in Eq. (1.1), $F_{+}$and $F_{\times}$are the detector beam patterns for the plus and cross polarisations of the gravitational waves, $\psi$ is the wave polarisation angle, and $\iota$ is the angle between the rotation axis of the pulsar and the line-of-sight. For a gravitational wave signal impinging on the Earth the signal arrival time at the detector, $t$, will be modulated by Doppler, time delay and relativistic effects caused by the motions of the Earth and other bodies in the solar system, so we want a reference frame which will not be affected by these. In general, for isolated pulsars ${ }^{2}$, such a frame is the solar system barycentre (SSB). To convert from $t$ to the time at the SSB, $t_{b}$, we must include a series of time correc-

\footnotetext{
2 assuming negligible proper motion and acceleration within a globular clusters.
} 
tions,

$$
t_{b}=t+\delta t=t+\frac{\mathbf{r} \cdot \hat{\mathbf{n}}}{c}+\Delta_{E_{\odot}}+\Delta_{S_{\odot}},
$$

where $\mathbf{r}$ is the position of the detector with respect to the solar system barycentre (SSB), $\hat{\mathbf{n}}$ is the unit vector pointing to the pulsar, $\Delta_{E_{\odot}}$ is the special relativistic Einstein delay, and $\Delta_{S \odot}$ is the general relativistic Shapiro delay (see Ref. 7] for definitions of these delay terms). For isolated pulsars we can therefore set $T=t_{b}$ in Eq. (1.1). For pulsars in binary systems there will be additional time delays as discussed in

In this paper we describe the addition of such binary system time delays with respect to current searches for gravitational waves from known pulsars. In a seperate issue we also discuss how pulsar timing noise can cause deviations from the simple phase model in Eq. 1.1 and how to account for this in the analysis.

\section{SEARCH METHOD}

Searches for gravitational waves from a selection of known pulsars have been performed using data from the LIGO [10] and GEO 600 [11] gravitational wave detectors from the four science runs (S1-4), which have taken place since late 2002 [12, 13, 14]. The search method used is outlined briefly here, but is described more fully in Refs. [8, 9].

All current data from these detectors is sampled at $16384 \mathrm{~Hz}$, giving a range of $0-8192 \mathrm{~Hz}$ available for searches, although in practice this is limited to the most sensitive region of the detectors between $\approx 50-4000 \mathrm{~Hz}$. The rotation frequencies of known pulsars are, of course, known very precisely from radio and/or X-ray observations, so the vast majority of this frequency space is redundant and the speed of any search can be increased by removing it. Knowledge of the pulsar parameters allows us to perform a complex heterodyne on the data, using the precise pulsar phase evolution, and down-sample it to $\frac{1}{60} \mathrm{~Hz}$. Following this heterodyne a Bayesian parameter estimation for the unknown pulsar parameters $h_{0}, \psi$, $\cos \iota$ and $\phi_{0}$ can be performed on the massively reduced data set.

The method relies on the accuracy of the signal model, and it is very important that the phase evolution in the heterodyne is sufficiently good. Any drift from the true pulsar signal phase could nullify the search if it becomes too severe. This paper will discuss how to ensure that this phase model is sufficiently accurate for pulsars in binary systems and those strongly affected by timing noise. Most recently, these methods have been used in Ref. 14] to obtain limits on the gravitational wave emission from 78 pulsars using data from the LIGO and GEO 600 S3 and S4 runs.

\section{PULSARS IN BINARY SYSTEMS}

Of the 1627 pulsars in the ATNF catalogue, 124 are in binary systems. The first of these to be discovered was the highly relativistic pulsar PSR J1915+1606 found by Hulse and Taylor in 1974 [15]. Of these 124 pulsars, 98 have spin frequencies greater than $25 \mathrm{~Hz}$ (out of a total 163 isolated and binary pulsars with frequencies greater than $25 \mathrm{~Hz}$ ), and therefore gravitational wave frequencies $>50 \mathrm{~Hz}$, putting them into the sensitive band of the LIGO detectors. Indeed, for the reason stated in 9 , the majority of millisecond pulsars are in binary systems.

\section{A. Pulsar timing}

A brief discussion on how pulsar timing information is obtained is relevant here. The majority of pulsars have been both discovered and monitored in radio. Pulsar surveys, discussed in more detail in Ref. 16], use Fourier transform methods to look for periodic signals in the radio data, taking into account the effect of interstellar dispersion across the receiver band. Once the pulsar period has been determined, the radio time series data can be folded with this cadence to build up the signal-to-noise ratio of a mean pulse. Once a stable pulse is obtained ${ }^{3}$ the time of arrival (TOA) can be measured at the peak of the pulse. These pulse times can then be used to extract more precise information about the pulsar parameters, including its position and frequency parameters.

The most prevalent tool used to fit timing measurements is the TEMPO software suite [17], however others have been developed, including PSRTIME [18] at Jodrell Bank Observatory. TEMPO requires precise solar system ephemerides, containing the positions and velocities of the major solar system bodies, to convert TOAs at a detector to the rest frame of the pulsar. It computes the pulsar phase at each TOA, $\phi\left(T_{i}\right)$, over the range of pulsar parameters $(\alpha, \delta, \nu, \dot{\nu}$, etc $)$, and uses a $\chi^{2}$ goodness-of-fit statistic to determine the best model via minimization. A starting point for the fit is obtained through a rough knowledge of the position and frequency from the initial discovery, but it can still be quite complex as there can be many other parameters that could be contributing. Below it is seen how a pulsar in a binary system requires a complex model with many more parameters than an isolated object.

\section{B. Binary pulsar timing}

The majority of pulsars within the ground-based gravitational wave frequency band are in binary systems, so

\footnotetext{
${ }^{3}$ Individual pulses can vary in shape, but the summation of many gives a generally stable pulse shape.
} 
we must consider the effects of binary timing carefully. Techniques to create filters to match a binary signal (in the frequency domain) have been described before, for example in Ref. [19], and these have been applied to the search for gravitational waves from the neutron star in Sco X-1 [20], but there has been no equivalent treatment in the time domain, suitable for the search outlined above. Eq. (1.3) shows the timing corrections needed to take account of Doppler and relativistic delays of a signal and transform it to the SSB. Any constant Doppler delays from the pulsar's actual motion relative to the SSB are unimportant, and the SSB frame can be considered as the rest frame of the pulsar. For a pulsar in a binary system however, its motion within the system will need to be taken into account with a transform from the binary system barycentre to the pulsar proper time.

The basic transformation and binary models below are summarised in Ref. [7] and used in the pulsar timing program Tempo [17]. The transformation from SSB time $t_{b}$ to pulsar proper time $T$ follows the form of Eq. (1.3) and is

$$
t_{b}-t_{0}=T+\Delta_{\mathrm{R}}+\Delta_{\mathrm{E}}+\Delta_{\mathrm{S}}+\Delta_{\mathrm{A}},
$$

where $\Delta_{R}$ is the Roemer time delay giving the propagation time across the binary orbit; $\Delta_{\mathrm{E}}$ is the Einstein delay and gives gravitational redshift and time dilation corrections; $\Delta_{\mathrm{S}}$ is the Shapiro delay which gives the gravitational propagation delay due to the signal propagation through the curved space-time of the companion; and $\Delta_{\mathrm{A}}$ is the aberration delay caused by the pulsar's rotation.

The majority of binary orbits can be well-modelled as Keplerian. Keplerian orbits are defined by five parameters, $T_{0}$ - the time of periastron (closest approach in the binary orbit); $\omega$ - the longitude of periastron; $P_{b}$ - the orbital period; $e$ - the orbital eccentricity (where $e=\sqrt{\left(1-b^{2} / a^{2}\right)}$ and $a$ and $b$ are the semi-major and semi-minor axis of the orbital ellipse respectively); and $x \equiv(a \sin i) / c$ is the projected semi-major axis, with $i$ being the orbital inclination. For some of the most extreme binary systems, with rapidly-spinning pulsars in tight orbits, the maximum (gravitational wave) orbital Doppler frequency shift can be up to $\sim 0.1 \mathrm{~Hz}$. For example, PSR J0737-3039A with $x=1.415$ light sec, $P_{b}=$ 0.10225 days and $\nu_{\mathrm{gw}}=88.11 \mathrm{~Hz}$ gives $\Delta \nu_{\mathrm{gw}}=0.089 \mathrm{~Hz}$.

We will now consider the three most commonly used models used to characterise the TOA of pulses from pulsars in binary systems.

\section{Blandford-Teukolsky model}

The Blandford and Teukolsky model 21] (BT) makes no assumptions about the correct theory of gravity. Instead, it assumes a simple Keplerian orbit with slow precession, into which additional relativistic effects have been added. Other phenomena can be taken into account through time derivatives of the four main orbital elements, excluding $T_{0}$. The BT model has been used to fit data for 53 of the binary pulsars with $\nu>25 \mathrm{~Hz}$, and is the most common model used. Exceptionally, one of these systems is modelled using the TEMPO model BT2P which accommodates three orbits, the first of which can be relativistic, but the second and third are Keplerian. The system is a multiple system, described in Ref. [22], in which three, or possibly four, planets orbit the pulsar. Although these additional orbits complicate the above equations, the standard BT model is sufficient for our purposes.

\section{Low eccentricity model}

The second most common model used in fitting radio observations of binaries is the low eccentricity model (called ELL1 in TEMPo) developed in Ref. [23]. It is used as a fit for pulsars in very low eccentricity orbits $(e \simeq 0)$ and is the appropriate model for 38 of our target pulsars with $\nu>25 \mathrm{~Hz}$. An almost circular orbit makes $T_{0}$ and $\omega$ nearly degenerate, so these parameters, along with $e$, are replaced with the non-covariant parameters of the time of the ascending node of the orbit $\left(T_{\mathrm{asc}} \equiv T_{0}-\omega P_{b} / 2 \pi\right)$ and the first and second Laplace-Lagrange parameters $\eta \equiv e \sin \omega$ and $\kappa \equiv e \cos \omega$. The time delays for this model are defined in 23] and TEMPo.

\section{Damour-Deruelle model}

The third most common model is that of Damour and Deruelle (DD) 24]. This model uses a method for solving the relativistic two-body problem to post-Newtonian order and is valid under very general assumptions about the nature of gravity in strong field regimes. It is useful for highly relativistic systems, although in only mildly relativistic systems this model should be no different to the BT model. There are seven pulsars with $\nu>25 \mathrm{~Hz}$ in the ATNF catalogue that use this model. This model is again summarised in [7].

\section{Comparison with TEMPO}

The above three models are all implemented in the pulsar timing software package TEMPO. In our search for gravitational waves from binary systems we also require these additional time corrections to correctly calculate the phase of the pulsar for heterodyning. Code to calculate the binary time delays for each model has been adapted from the TEMPO counterparts and is available under CVS in the LIGO Algorithm Library (LAL) repository [25]. Some consistency tests have been performed between the two codes, which are described below. It is acknowledged that TEMPO has some uncertainties in its timing models due to simplifying assumptions, and these limit its accuracy to $\sim 100 \mathrm{~ns}$. These effects are dis- 
cussed in Refs. [26, 27], but as errors at this level have no significant impact on our search we will neglect them.

\section{PSR J1012+5307}

We performed a validation check on the new LAL code by demodulating radio data from a known pulsar. With no known gravitational calibrators, this is a crucial step in the development of any gravitational wave detection code. A set of TOAs for PSR J1012+5307 obtained with the Effelsberg $100 \mathrm{~m}$ radio telescope in Bonn, Germany, was kindly supplied by Michael Kramer for this purpose. This pulsar has the second most circular orbit known and is therefore fitted by the ELL1 model. The data intermittently spanned just over 5 years from $2^{\text {nd }}$ January 2000 to $12^{\text {th }}$ February 2005 and comprised TOAs (in Modified Julian Date format, where MJD = Julian Date-2400000.5), together with the TEMPO-derived pulsar parameters (Table I). Some minor transformations

TABLE I: The parameters of PSR J1012+5307. Values are quoted with $1 \sigma$ errors on the final digit in brackets.

\begin{tabular}{r|l}
\hline \hline PSR J1012+5307 \\
$\alpha$ & $10^{\mathrm{h}} 12^{\mathrm{m}} 33^{\mathrm{s}} .43368(1)$ \\
$\delta$ & $53^{\circ} 07^{\prime} 02^{\prime \prime} .5880(2)$ \\
PMRA & $2.38(3) \mathrm{mas} / \mathrm{yr}$ \\
PMDEC & $-25.35(5) \mathrm{mas} / \mathrm{yr}$ \\
$\nu$ & $190.267837621884(9) \mathrm{Hz}$ \\
$\dot{\nu}$ & $-6.2022(2) \times 10^{-16} \mathrm{~Hz} / \mathrm{s}$ \\
$\ddot{\nu}$ & $2.0(3) \times 10^{-27} \mathrm{~Hz} / \mathrm{s}^{2}$ \\
Frequency epoch & MJD 50700 \\
Dispersion measure & $9.0233(7) \mathrm{cm}^{-3} \mathrm{pc}$ \\
Observing Frequency & $1408.6 \mathrm{MHz}$ \\
Binary model & ELL1 \\
$x$ & $0.581817(1) \mathrm{s}$ \\
$P_{b}$ & $0.6046727136(2)$ days \\
$T_{\text {asc }}$ & MJD 50700.0816289(4) \\
$\eta$ & $7(4) \times 10^{-7}$ \\
$\kappa$ & $-1(40) \times 10^{-8}$ \\
\hline \hline
\end{tabular}

are necessary to convert TOAs measured at the telescope to the GPS time stamps used in gravitational wave data analysis software. First the raw TOAs are corrected for the drifts between the hydrogen maser clock at Effelsberg and coordinated Universal Time of the National Institute of Science and Technology UTC(NIST) reference. This correction (supplied with the data) was typically a few microseconds. The difference between UTC(NIST) and UTC has been less than \pm 100 ns since $6^{\text {th }}$ July, 1994 [28] and was neglected for this work. The conversion between the time scales therefore becomes

$$
t_{\mathrm{GPS}}=\left(t_{\mathrm{UTC}(\mathrm{MJD})}-44244 \text { days }\right) \times 86400 \mathrm{~s}+L,
$$

where the 44244 corresponds to the MJD of the GPS time epoch $\left(1^{\text {st }}\right.$ January, 1980) and $L$ is the accumulated number of leap seconds included in the definition of UTC. For the time-span of these TOAs, $L=13$.
The TOAs can now be corrected for interstellar dispersion time delay [16]

$$
\Delta t_{\text {disp }}=4.149 \times 10^{3} \mathrm{MHz}^{2} \mathrm{pc}^{-1} \mathrm{~cm}^{3} \mathrm{~s} \times \mathrm{DM} / f^{2} \mathrm{~s},
$$

where DM is the dispersion measure in $\mathrm{cm}^{-3} \mathrm{pc}$ and $f$ is the radio observation frequency in $\mathrm{MHz}$ (see Table $\prod$ for values). This correction is subtracted from the TOAs to give observations at infinite frequency with no dispersion.

One of the major differences between our binary time domain code and the TEMPo code is the time system used. All epochs in TEMPO are defined as MJD Barycentric Dynamical Time (TDB - a timescale generally used for ephemerides referenced to the solar solar barycentre) whereas the general reference time for our gravitational wave data is GPS time. Epochs therefore have to be converted to GPS time on the TDB timescale. This TDB timescale is related to Terrestrial Time (TT - formerly Terrestrial Dynamical Time TDT), which represents a time consistent with relativity for an observer on the Earth's surface, by a small factor, $\mathrm{TDB}=\mathrm{TT}+\delta t$, no greater than a couple of milliseconds and given by

$$
\delta t=0.001658 \mathrm{~s} \times \sin \Phi+0.000014 \mathrm{~s} \times \sin 2 \Phi,
$$

where $\Phi=357.53^{\circ}+0.98560028^{\circ}(\mathrm{MJD}-51544.5)$ is the mean anomaly, or phase, of the Earth's orbit at the given Modified Julian Date [29]. TT is offset from International Atomic Time (TAI), so that $\mathrm{TT}=\mathrm{TAI}+$ 32.184 seconds $^{4}$. The conversion thus goes $t_{\mathrm{TDB}(\mathrm{GPS})}=$ $\left(t_{\mathrm{TDB}(\mathrm{MJD})}-44244\right)-51.184-\delta t$, where the $51.184 \mathrm{~s}$ comprises the $32.184 \mathrm{~s}$ difference between TT and TAI and 19 second difference between TAI and GPS.

The LAL code to calculate the SSB time delay uses the pulsar's position, the telescope position and a solar system ephemeris 30] and was used for each pulsar TOA to correct to the time at the SSB. This code was written by Curt Cutler and has been independently tested against TEMPO [8, 12] showing no more than $4 \mu$ s difference between the two. For a pulsar with a gravitational wave frequency at $1 \mathrm{kHz}$ a $4 \mu$ s timing error would give a phase offset between any signal and our model template of 0.025 rads. This would reduce our sensitivity to a signal by of order $1-\cos 0.025 \approx 3 \times 10^{-4}$, which is negligible.

Once corrected to the SSB the TOAs are further corrected to the pulsar proper time by calculating the time delays in the binary system using the binary system parameters (see Table【). The binary and solar system time delays for a selection of TOAs covering part of the binary orbit are shown in Fig. 1 .

Once these corrections to the TOAs are applied we can compare the LAL barycentring codes with TEMPO by inserting the TEMPO-derived pulsar parameters into the

\footnotetext{
4 There are many definitions of time used in astronomy and very careful attention of which one is being used and how to convert between them is essential when high precision timings are being made. A good guide for these is [29].
} 

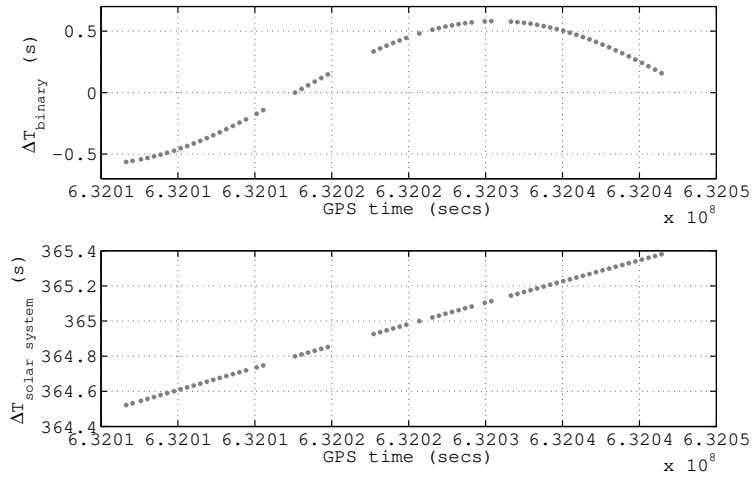

FIG. 1: The binary and solar system time delays calculated for PSR J1012+5307 over a part of a binary orbit.

LAL barycentring routines and examining the predicted phase at the corrected TOAs. TOAs converted incorrectly by the barycentring codes would show up as a phase drift. The phase at each TOA was calculated using the supplied frequency and frequency derivatives in Eq. (1.1), with $\phi_{0}=0$ and the frequency epoch as $t_{0}$. Fig. 2 demonstrates the serious effect of neglecting these binary time delays. In contrast, Fig. 3 shows how

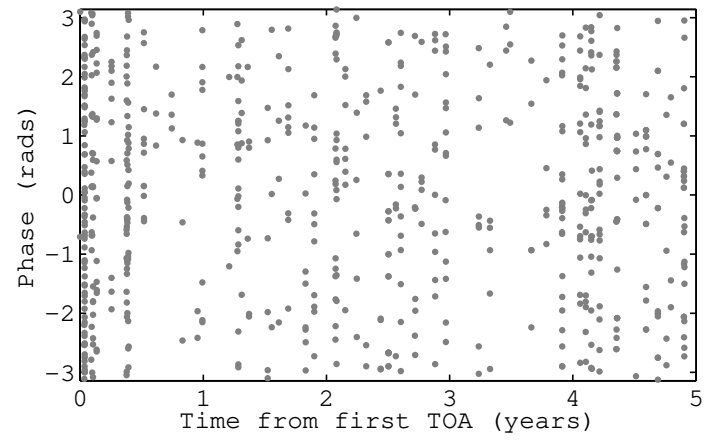

FIG. 2: The modulus of the pulsar phase at each TOA over a 5 year period with no binary time delays included.

the TOAs barycentred using our code stay well in phase over the observation time when the binary delays are included, with a residual phase slope of $\sim 0.04 \mathrm{rads} / \mathrm{yr}$. A yearly periodicity is also present possibly showing up the slight difference in the LAL solar system barycentring code and TEMPo, although these effects are at a very low level. Several points clearly show large phase residuals and correspond to times when the level of noise on the TOA measurements was high.

The parameters for PSR J1012+5307 were generated using the ELL1 model, so the above test only checked the ELL1 code. We can also check the two other models by converting $T_{\text {asc }}$ to $T_{0}$ and the Laplace-Lagrange parameters $\kappa$ and $\eta$ to $e=\sqrt{\left(\kappa^{2}+\eta^{2}\right)}$ and $\omega$. This pulsar

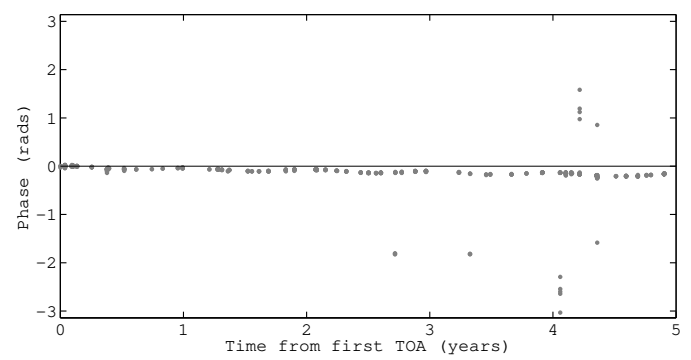

FIG. 3: The modulus of the pulsar phase at each TOA over a 5 year period corrected for the binary delay.

has a low eccentricity, so $T_{0}$ can be set equal to $T_{\text {asc }}$ and $e$ and $\omega$ set to zero for practical purposes. Doing this we can again produce the phase plots for the BT and DD models (Fig. (4). The phase is again well described
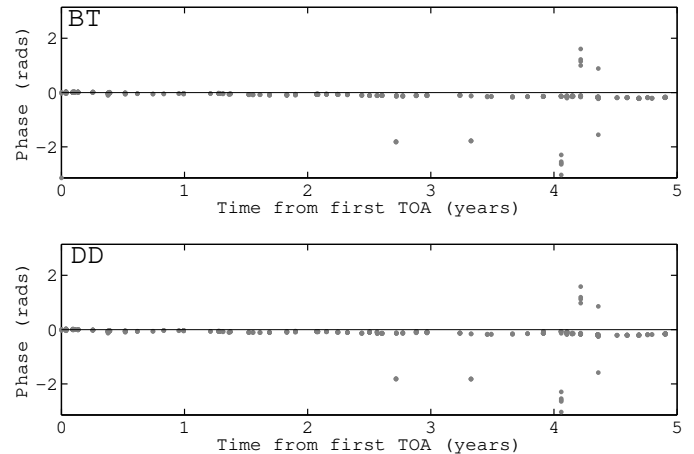

FIG. 4: The modulus of the pulsar phase at each TOA over a 5 year period for the BT and DD models, corrected for the binary delay.

for these two models, with the slope and periodicity still present. This suggests that the slope and periodicities are not caused by the binary timing correction code (as each model is independent), but may be a results of slight errors in the other timing corrections, the solar system barycentring code, the ephemerides, or the parameters used.

The effects of inaccuracies in these binary parameters can be shown by offsetting one from its true value. In Fig. [5 the true value of the $T_{\text {asc }}$ parameter has been offset by 5,10 and 20 seconds and the phase at each TOA recalculated. Even for a $5 \mathrm{~s}$ mismatch we start to depart from the true phase by up to 0.5 radians. This would not be disastrous for the analysis but would degrade the search. For a $20 \mathrm{~s}$ offset we start to see phase errors of up to 2 radians. At this level, all sensitivity is lost. In real analyses as in Ref. [14 the effect of any errors on the parameters, as to whether they could cause our heterodyne phase to depart significantly from the signal phase, is thoroughly checked. 


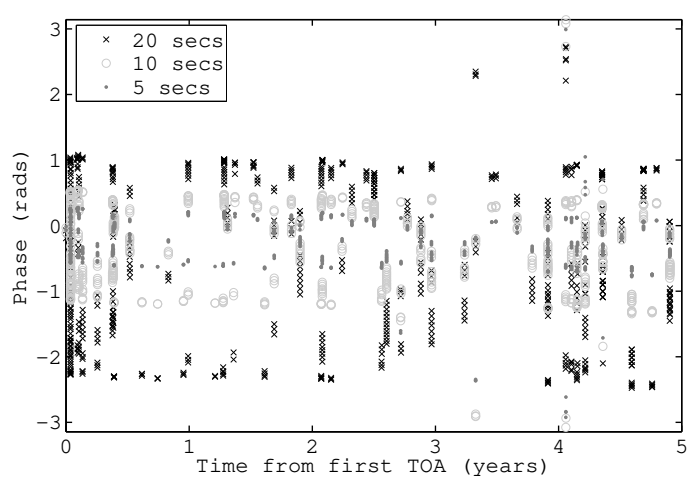

FIG. 5: The modulus of the pulsar phase at each TOA over a 5 year period with $T_{\text {asc }}$ offset from its true value.

\section{Direct check against TEMPO}

In common with the solar system barycentring code [8, 13], the binary timing code was tested directly against Tempo. Tempo can be run in predictive mode, to use a set of pulsar parameters to predict the pulsar phase over a period of time. This predicted phase can be then be compared with that calculated using our binary timing code. This was done for each model with a set of 100 randomly generated binary pulsar systems over a period of 100 days. The detector location was set to be at the SSB, so the solar system time delay errors would not be included. Histograms of the time residuals between the codes are shown in Fig. 6 for each model. These show that the time difference between the two codes is generally less than $\pm 1 \mu \mathrm{s}$, which is sufficiently small to ensure any signal and template remain in phase over the frequency range considered.

\section{THE PROBLEM OF TIMING NOISE}

A rather different issue that if unaddressed could potentially cause problems for our analysis is that of timing noise.

Pulsars are generally very stable over periods of several days, but there are phenomena which can cause deviations in this timing stability. With the very high accuracy of pulsar timing any random timing irregularities will start to become evident. These phenomena show up as glitches and timing noise. Timing noise has been known about since the early days of pulsar observations and represents a random walk in phase, frequency or frequency derivative of the pulsar about the regular spindown model given in Eq. (1.1) [31]. If our search method cannot take account of these phase deviations they could reduce our sensitivity or even invalidate the search. It is therefore important to see firstly if timing noise produces large enough phase deviations to cause a problem for our method and if so how it can be countered.

The strength of this effect has been quantitatively defined in Ref. [31] as the activity parameter $A$, as referenced to that of the Crab pulsar, and in Ref. 32 as the stability parameter $\Delta_{8}$. There is however no real consensus on how to quantitatively define a measure of the level of timing noise, with the magnitude and sign of $\ddot{P}$ maybe providing the measure which includes the least other assumptions. A thorough study of timing noise, comparing and contrasting the various measures used, is given in Ref. [33] (also see Refs. [34, 35]). There is a definite correlation between these parameters ( $A$ and $\left.\Delta_{8}\right)$ and the pulsar's spin-down rate, therefore possibly the pulsar's age. Young pulsars, like the Crab pulsar, generally show the most timing noise activity.

The Crab pulsar is the youngest known pulsar targeted by current gravitational wave detectors and it is important to considered how timing noise may be countered for this pulsar. A method was first proposed in Ref. 36] and has been used in the analysis of Abbott et al. [13], but has not previously been described in detail. A brief look into the effects of timing noise for other pulsars will be discussed later.

\section{A. Timing noise in the Crab pulsar}

The pulsar $(\mathrm{J} 0534+2200)$ in the Crab nebula (M1) has undergone intense study since its discovery in 1968. Its parameters are given in Table II. It has an observed

TABLE II: The parameters of the Crab pulsar as calculated from the Jodrell Bank monthly ephemeris 39].

\begin{tabular}{c|l}
\hline \hline \multicolumn{2}{c}{ PSR J0534+2200 } \\
\hline Right ascension $\alpha$ & $05^{\mathrm{h}} 34^{\mathrm{m}} 31^{\mathrm{s}} .973$ \\
Declination $\delta$ & $22^{\circ} 00^{\prime} 52^{\prime \prime} .06$ \\
proper motion in $\alpha$ & $-13 \mathrm{mas} / \mathrm{yr}$ \\
proper motion in $\delta$ & $7 \mathrm{mas} / \mathrm{yr}$ \\
Position epoch & $\mathrm{MJD} 40675$ \\
$\nu$ & $29.7670971390 \mathrm{~Hz}$ \\
$\dot{\nu}$ & $-3.72633 \times 10^{-10} \mathrm{~Hz} / \mathrm{s}$ \\
$\ddot{\nu}$ & $1.1429 \times 10^{-20} \mathrm{~Hz} / \mathrm{s}^{2}$ \\
Frequency epoch & $\mathrm{MJD} 53993$ \\
Distance & $2.0 \mathrm{kpc}$ \\
\hline \hline
\end{tabular}

age of 951 years (the formation of the Crab nebula is associated with a supernova observed in AD 1054) and a spin-down age of $-\nu / 2 \dot{\nu}=P / 2 \dot{P}=1250$ years. Analyses of long-term timing observation of the Crab pulsar are given in Refs. [37, 38]. These analyses show some of the timing features which make the Crab pulsar such an interesting object: the timing noise and glitches.

Since 1982 there has been a regular monitoring program of the Crab pulsar at Jodrell Bank Observatory, and timing ephemerides from this are publicly available online [39]. The ephemeris gives the pulsar frequency 

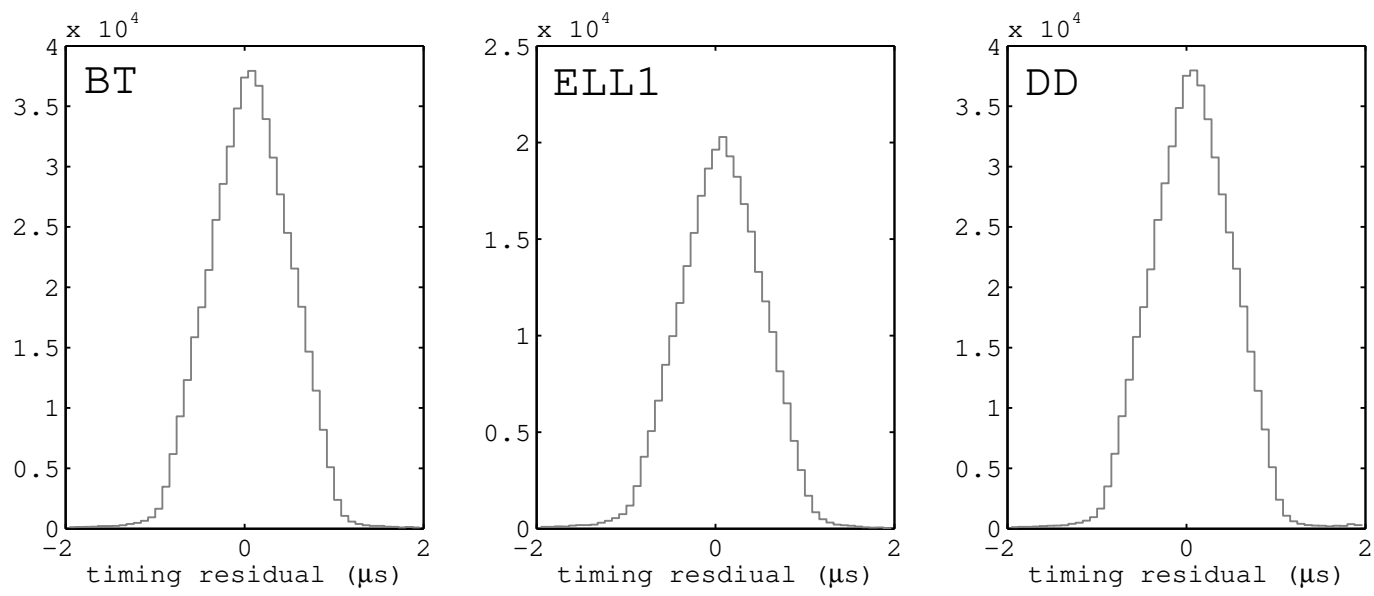

FIG. 6: Timing residuals between the pulsar phase as predicted by TEMPO and that computed with our binary code for 100 random pulsars for each binary model.

and frequency derivative and associated errors, and the associated epoch. The epochs, generally given on the $15^{\text {th }}$ of each month, represent the time of the peak of the first pulse after midnight on that day. They therefore represent zero of modulus phase of the electromagnetic pulse. Notes are given in the event of a timing irregularity or glitch being observed. The Crab's timing noise can be clearly seen in the ephemeris once a best-fit quadratic timing model (Eq. (1.1)) has been subtracted (see Fig. 7). The section of data used was chosen to be free of glitches as these can be much larger than any timing noise frequency deviations. Fig. 7 compares well with that given

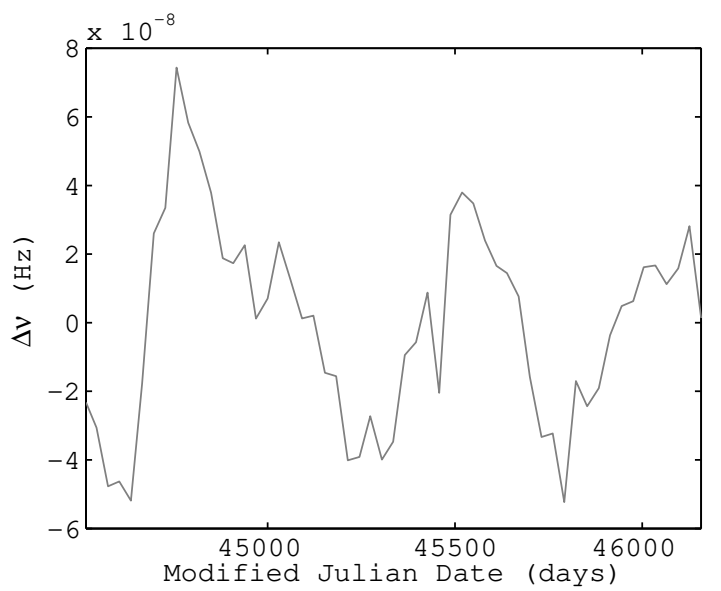

FIG. 7: The timing noise in the frequency of the Crab pulsar after removing a quadratic fit to the frequency as given in the Jodrell Bank ephemeris.

in Ref. [37], although some difference can be expected due to the different lengths of data and epochs used in the fitting. It can be seen that on scales of several months there is quite a large variation in the timing residual. It is shown in Ref. [37] that on smaller time scales the variation is far smoother. It is important to consider whether timing noise at this level will cause a loss in sensitivity in any search for gravitational wave emission from the Crab pulsar. Jones [40] constructs a decoherence timescale, $T_{\text {decoherence}}$, defined as the time over which the timing noise will cause the phase to deviate by 1 radian from the second order Taylor expansion of phase. This makes use of the "activity parameter" and is calculated to be $\sim 2.6 \mathrm{yr}$ for the Crab pulsar. Though useful as an indicator, this statistic does not take account of permanent changes to spin-down caused by glitches or other secular variations in the pulsar.

Although it is reasonable that a third order fit to the entire data would improve the model, there is no need. The Crab pulsar ephemeris provides timing every month, which is sufficient to track the phase excursions in the timing noise. All that is necessary is that these be interpolated between the ephemeris times. By using the phase, frequency and frequency derivative for each entry in the ephemeris as boundary conditions to a set of simultaneous equations the full phase evolution between each month can be calculated, giving a fifth order polynomial,

$$
\begin{gathered}
\phi_{5^{\text {th }}}(T)=\phi_{0}+2 \pi\left\{\nu_{0}\left(T-t_{0}\right)+\frac{1}{2} \dot{\nu_{0}}\left(T-t_{0}\right)^{2}+\right. \\
\left.\frac{1}{6} \ddot{\nu}_{0}\left(T-t_{0}\right)^{3}+\frac{1}{24} \dddot{\nu}_{0}\left(T-t_{0}\right)^{4}+\frac{1}{120} \dddot{\nu}_{0}\left(T-t_{0}\right)^{5}\right\}
\end{gathered}
$$

Indeed, for much of the time even this method is unnecessarily complicated and a simple linear interpolation between months would be sufficient.

These corrections can be included in the method detailed in $₫ \amalg$ as an extra heterodyne step [36]. The initial heterodyne (described in $\$$ III), uses a third order fit to the the phase with values of $\nu$ and $\dot{\nu}$ taken from the ephemeris at the closest time before the timestamp on the data to be analysed, and $\ddot{\nu}$ taken from the ATNF 
pulsar catalogue value given in Table [II Then, assuming that any gravitational wave signal would show the same timing noise (see Refs. 40] and 14 for discussions of this), we apply a second heterodyne to the data $B_{k}$ using the phase difference between Eqs. (1.1) and (4.1)

$$
B_{k}^{\prime}=B_{k} e^{-i 2\left[\phi_{5} \text { th }(T)-\phi(T)\right]},
$$

where the factor of two in the phase is due to the gravitational wave frequency being twice the spin frequency. This step can be performed on the data after downsampling as the rate of change of this phase difference will be very low.

The effect of this extra heterodyne can be seen in search for a signal from the Crab in the S2 data. This science run of the LIGO interferometers lasted approximately two months and overlapped three entries in the Crab pulsar ephemeris. The S2 run started on $14^{\text {th }} \mathrm{Feb}$ 2003, so values of the frequency and spin-down used in the initial heterodyning were chosen to be those given in the first ephemeris entry prior to the run (15th Jan 2003). The second derivative was set to be that taken from the ATNF catalogue. The values are shown in Table III Once the data were produced the ephemeris values were

TABLE III: The parameters used in the initial heterodyne stage of the Crab pulsar analysis for S2.

\begin{tabular}{c|l}
\hline \hline \multicolumn{2}{c}{ PSR J0534+2200 } \\
\hline$\nu$ & $29.8102713888 \mathrm{~Hz}$ \\
$\dot{\nu}$ & $-3.736982 \times 10^{-10} \mathrm{~Hz} / \mathrm{s}$ \\
$\ddot{\nu}$ & $1.2426 \times 10^{-20} \mathrm{~Hz} / \mathrm{s}^{2}$ \\
Frequency epoch & GPS 726624013 \\
\hline \hline
\end{tabular}

used to calculate the phase given in Eq. (4.1). The difference between the initial heterodyne phase and the $5^{\text {th }}$ order phase is shown in Fig. 8. This phase difference is used in the extra heterodyne to remove the variation. It can be seen in Fig. 8 how a linear fit between ephemeris values would be acceptable for these times, with only small deviations in phase from the fifth order fit. The black crosses in Fig. 8 provide the first step in checking the code used for the extra heterodyne stage. The red points represent the phase difference used in our extra heterodyne step (Eq. (4.2)) to heterodyne each S2 data point as calculated using our code, whereas the black crosses just show the phase difference between the initial heterodyne and the individual Crab pulsar ephemeris data points. The fact that these overlap provides a check that the heterodyne code is producing the correct phase difference.

If we simulate a signal from the Crab pulsar over the period of $\mathrm{S} 2$, with parameters $h_{0}=0.5, \phi_{0}=0.0, \psi=0.0$ and $\iota=\pi$, we can see how including a timing noise heterodyne step affects the parameter estimation. Fig. 9 shows the extracted probability distribution functions (pdfs) of $h_{0}$ and $\phi_{0}$ for the signal with and without the

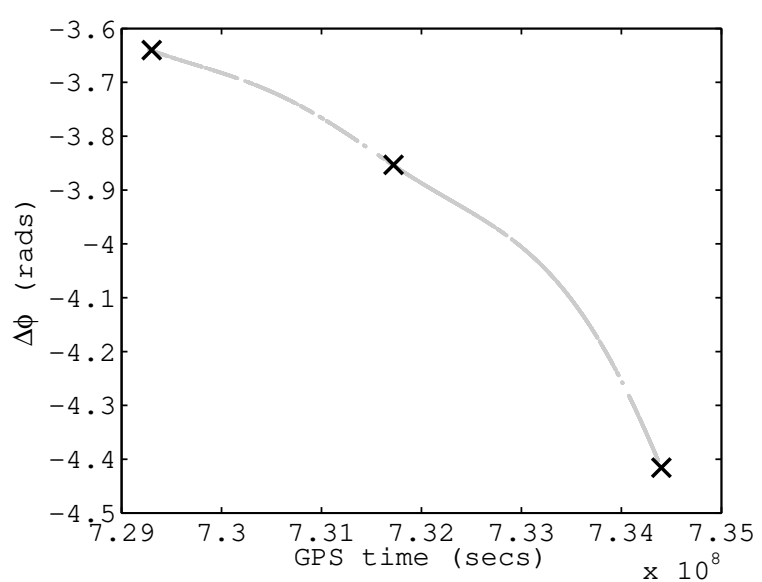

FIG. 8: The grey points show the phase difference between that used in the initial heterodyne and that interpolated from a fifth order fit to the ephemeris. The black crosses show just the phase difference between the initial heterodyne and the individual ephemeris values.

timing noise removed. There is very little difference between the amplitudes for the two cases because the slope of the phase difference $\Delta \phi$ are not too steep over the period of S2. However, the extracted value of the phase
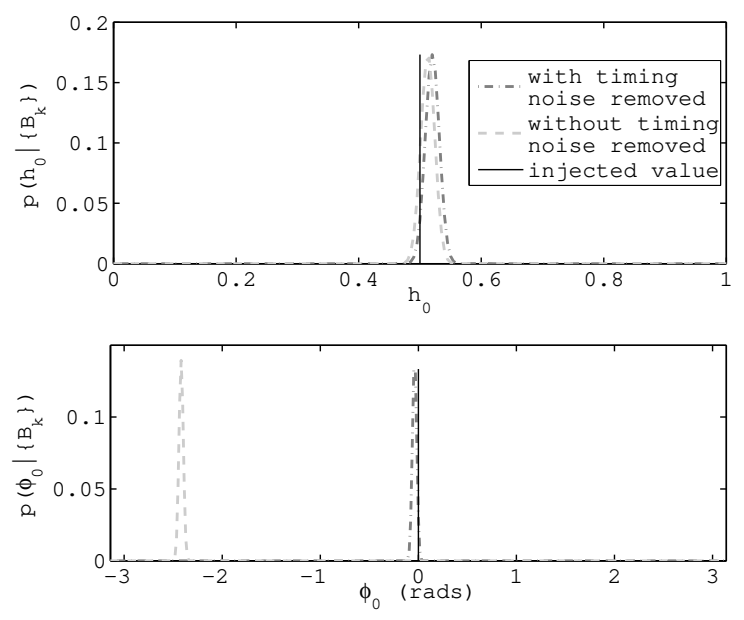

FIG. 9: The extracted pdfs for $h_{0}$ and $\phi_{0}$ for a simulated signal from the Crab pulsar over the period of S2 with and without timing noise removed.

is strongly affected, mainly due to the the phase offset between the start of S2 and the epoch of the initial heterodyne parameters seen in Fig. 8 .

We can simulate a Crab pulsar signal and analyse it with and without the timing noise heterodyne step over longer periods than just S2 to show its importance. The same process as above has been carried out over the period of the S3 run, using the same initial heterodyne pa- 
rameters and pulsar injection parameters. The extracted pdfs are shown in Fig. 10 and demonstrate that without the extra heterodyne the signal is completely lost. The
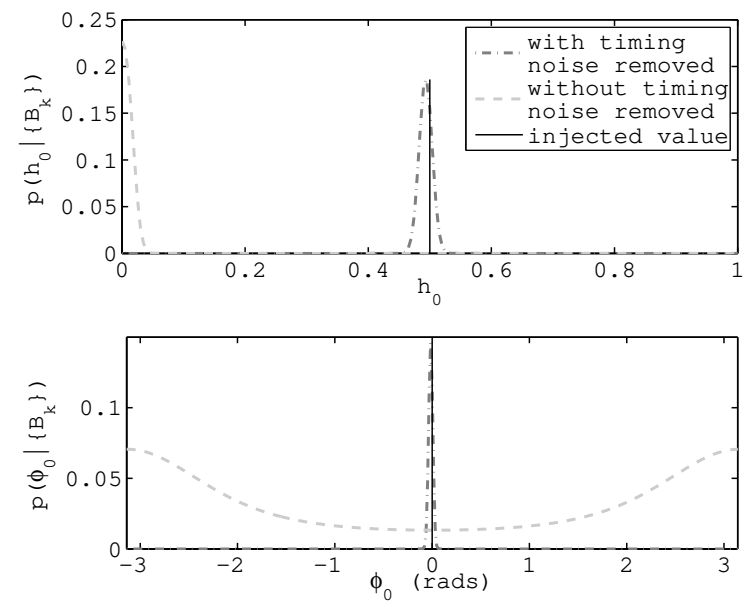

FIG. 10: The extracted pdfs for $h_{0}$ and $\phi_{0}$ for a simulated signal from the Crab pulsar over the period of S3 with and without performing the extra heterodyne.

fact that this signal is not seen without the extra heterodyne may appear at odds with the approximate 2.6 year decoherence time stated above, as S3 was only about 8 months after S2. However, the initial heterodyne values used were still those from the Crab ephemeris closest to the start of S2 and not those from a more general fit to the data over an extended period, as was used to calculate $T_{\text {decoherence }}$, so it is not the timing noise causing the decoherence in this case, but badly chosen initial heterodyne parameters. This does however demonstrate the importance of having well-defined heterodyne parameter for all pulsars. If we perform a fit to the Crab pulsar ephemeris over the whole of 2003 (see Table IV for fit values), when the S2 and S3 runs took place, we can again check the impact of the timing noise in S3 (see Fig. 11). We see

TABLE IV: The parameters of the Crab pulsar for a fit to second order in frequency over the period of 2003 using monthly ephemeris data.

\begin{tabular}{c|l}
\hline \hline \multicolumn{2}{c}{ PSR J0534+2200 } \\
\hline$\nu$ & $29.81027139567395 \mathrm{~Hz}$ \\
$\dot{\nu}$ & $-3.73698 \times 10^{-10} \mathrm{~Hz} / \mathrm{s}$ \\
$\ddot{\nu}$ & $1.07086 \times 10^{-20} \mathrm{~Hz} / \mathrm{s}^{2}$ \\
Frequency epoch & GPS 726624013.0597030 \\
\hline \hline
\end{tabular}

that, with an extended fit for the Crab parameters, timing noise makes little difference over S3, although a slight phase offset is present.
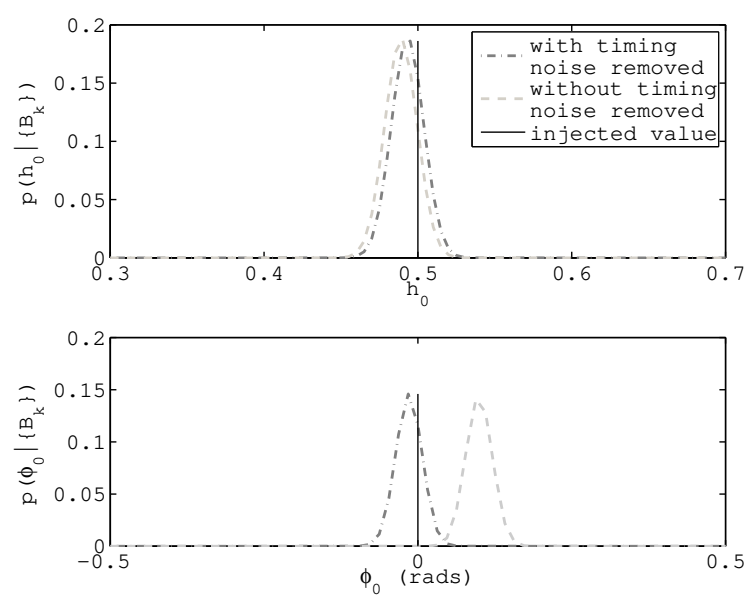

FIG. 11: The extracted pdfs for $h_{0}$ and $\phi_{0}$ for a simulated signal from the Crab pulsar over the period of S3 with and without timing noise removed for initial heterodyne values obtained from a fit to the ephemeris over 2003 (see Table IV].

\section{B. Timing noise in other pulsars}

For the majority of pulsars timing noise is most prominent in the second derivative of frequency, but for millisecond pulsars this value is often so small as to be unmeasurable. For these pulsars, the value of the $\Delta_{8}$ parameter can be used to estimate the cumulative phase contribution of timing noise via the empirical relationship between $\dot{P}$ and $\Delta_{8}$ given in Ref. [32]. Abbott et al. 11. used this as a test of the coherence of the pulsars phases over the periods of the LIGO and GEO 600 S3 and $\mathrm{S} 4$ data runs. The generally small values of $\ddot{\nu}$ for the majority of other pulsars suggest that there is not significant timing noise.

There is however, one known interesting target pulsar within our frequency range that has similar properties to the Crab pulsar. This pulsar, PSR J0537-6910, is young and has the second largest spin-down after the Crab pulsar, making it a very interesting candidate for our search. It is distant, lying in the Large Magellanic Cloud, and has so far only been observed in X-rays. It is also a prolific glitcher, showing high levels of timing noise [41]. As dedicated time on the Rossi X-ray Timing Explorer satellite is required to time this pulsar, it has not been observed as regularly as the Crab pulsar and a comprehensive ephemeris does not exist. A regularly updated ephemeris would however allow us to track the inter-glitch phase and perform an analysis similar to that of the Crab pulsar.

\section{CONCLUSIONS}

We have shown how to take account of time delays due to the motion of pulsars within binary systems in 
searches for gravitational waves. This is particularly important as a large number of the pulsars within the sensitivity band of current interferometric gravitational wave detectors are in binaries. However, as discussed in Ref. 14], we have demonstrated the importance of knowing the binary parameters and their covariances.

At present our search code contains the three main binary models described above. These are sufficient for the majority of pulsars, although TEMPO contains many more models which could be incorporated in the future if needed.

For the cases where large levels of timing noise are seen it is important that up-to-date parameters are obtained allowing us to track the rotational variations. Fortunately, the Crab pulsar is constantly monitored in radio and therefore has a very well-determined phase evolution. Other pulsars in our band are not so well monitored and this is especially pertinent if we want to target young pulsars (generally the best candidates for gravitational waves due to there large spin-down rates), because these will generally also be the most affected by timing noise. In particular, the young X-ray pulsar PSR J0537-6910 41] has high levels of glitch-induced timing noise, and an ability to regularly monitor this object would allow a more detailed study of this prime gravitational wave candidate.

\section{Acknowledgments}

The authors are very grateful to Michael Kramer for supplying pulsar timing information and useful discussions on its use. We would also like to acknowledge members of the LIGO Scientific Collaboration for useful advise during the writing of this manuscript. This work is to a large extent an extension of the work of Réjean Dupuis and to him we extend many thanks. This work has been supported by the UK Particle Physics and Astronomy Research Council.
[1] B. F. Schutz, Class. Quant. Grav 6, 1761 (1989).

[2] Australia Telescope National Facility Pulsar Catalogue http://www .atnf.csiro.au/research/pulsar/psrcat/

[3] R. N. Manchester et al, Astron. J. 129, 1993 (2005).

[4] D. R. Lorimer, Living Rev. Relativity 4(5) (2001). (cited on $12 / 12 / 06)$ http://www. livingreviews. org/lrr-2005-7

[5] E. P. J. van den Heuvel, Journal of Astrophysics and Astronomy 5, 209 (1984).

[6] P. Jaranowski, A. Królak, and B. F. Schutz, Phys. Rev. D 58, 63001 (1998).

[7] J. H. Taylor and J. M. Weisberg, Astrophys. J. 345, 434 (1989).

[8] R. J. Dupuis, PhD thesis, University of Glasgow (2004).

[9] R. J. Dupuis and G. Woan, Phys. Rev. D 72, 102002 (2005).

[10] B. Abbott et al, Nucl. Instrum. Meth. A517, 154 (2004), gr-qc/0308043.

[11] B. Willke et al, Class. Quantum Grav. 19, 1377 (2002).

[12] B. Abbott et al, Phys. Rev. D 69, 082004 (2004).

[13] B. Abbott et al, Phys. Rev. Lett. 94, 181103 (2005).

[14] B. Abbott et al, gr-qc/0702039.

[15] R. A. Hulse and J. H. Taylor, Astrophys. J. Lett. 195, L51 (1975).

[16] A. G. Lyne and F. Graham-Smith. Pulsar Astronomy. Cambridge Astrophysics Series. Cambridge University Press, Cambridge, 3rd edition, 2006.

[17] http://www.atnf.csiro.au/research/pulsar/tempo/

[18] http://www.jb.man.ac.uk/ pulsar/observing/progs/ps

[19] S. V. Dhurandhar and A. Vecchio, Phys. Rev. D 63, 122001 (2001).

[20] B. Abbott et al, gr-qc/0605028.

[21] R. Blandford and S. A. Teukolsky, Astrophys. J. 205, 580 (1976).

[22] A. Wolszczan et al, Astrophys. J. 528, 907 (2000).

[23] C. Lange et al, Mon. Not. R. Astron. Soc. 326, 274
(2001).

[24] T. Damour and N. Deruelle, Ann. Inst. Henri Poincaré A Phys. Théor. 44, 263 (1986).

[25] http://www.lsc-group.phys.uwm.edu/daswg/projects/lal.htm]

[26] G. Hobbs, R. T. Edwards and R. N. Manchester, Mon. Not. R. Astron. Soc. 369, 655 (2006).

[27] R. T. Edwards, G. Hobbs and R. N. Manchester, Mon. Not. R. Astron. Soc. 372, 1549 (2006). astro-ph/0607664.

[28] Bureau International des Poids et Mesures (BIPM) Circular $T$ http://www.bipm.fr/en/scientific/tai/

[29] Explanatory Supplement to the Astronomical Almanac, University Science Books (1992) http://www.cv.nrao.edu/ rfisher/Ephemerides/times.html.

[30] J. D. Giorgini et al., "JPL's On-Line Solar System Data Service", Bulletin of the American Astronomical Society 28(3), 1158 (1996). http://ssd.jpl.nasa.gov/?horizons

[31] J. M. Cordes and D. J. Helfand, Astrophys. J. 239, 640 (1980).

[32] Z. Arzoumanian et al., Astrophys. J. 422, 671 (1994).

[33] G. Hobbs, A. G. Lyne and M. Kramer, in preparation.

[34] G. Hobbs et al., Mon. Not. R. Astron. Soc. 353, 1311 (2004).

[35] G. Hobbs et al., Mon. Not. R. Astron. Soc. 360, 974 (2005).

[36] M. Pitkin and G. Woan, Class. Quantum Grav. 21, S843 (2004).

[37] A. G. Lyne, R. S. Pritchard, and F. Graham-Smith, Mon. rtime. Attontl R. Astron. Soc. 265, 1003 (1993).

[38] T. Wong, D. C. Backer, and A. G. Lyne, Astrophys. J. 548, 447 (2001).

[39] Jodrell Bank Crab Pulsar Monthly Ephemeris http://www.jb.man.ac.uk/ pulsar/crab.html

[40] D. I. Jones, Phys. Rev. D 70, 042002 (2004).

[41] F. E. Marshall et al, Astrophys. J. 603, 682 (2004). 